\title{
Ocorrência e diversidade de ácaros (Acari, Arachnida) associados a Tabebuia roseo-alba (Ridl.) Sand (Bignoniaceae), no município de São José do Rio Preto, São Paulo, Brasil ${ }^{1}$
}

\author{
Reinaldo José Fazzio Feres ${ }^{2}$, Marcos Roberto Bellini ${ }^{3} \&$ Denise de Cerqueira Rossa-Feres ${ }^{2}$
}

\author{
1 Parte do Programa BIOTA/FAPESP - O Instituto Virtual da Biodiversidade, http://www.biota.org.br \\ 2 Departamento de Zoologia e Botânica, Universidade Estadual Paulista. Rua Cristóvão Colombo 2265, Jardim Nazareth, \\ 15054-000 São José do Rio Preto, São Paulo, Brasil. E-mail: reinaldo@zoo.ibilce.unesp.br, denise@zoo.ibilce.unesp.br \\ ${ }_{3}^{3}$ Programa de Pós-graduação em Entomologia Agrícola, Universidade Estadual Paulista. Via de acesso Prof. Paulo Donato \\ Castellane, 14884-900 Jaboticabal, São Paulo, Brasil. E-mail: mrbellini@yahoo.com.br
}

\begin{abstract}
Occurrence and diversity of mites (Acari, Arachnida) associated with Tabebuia roseo-alba (Ridl.) Sand (Bignoniaceae), from São José do Rio Preto, São Paulo State, Brazil. This study reports the mite fauna associated with Tabebuia roseo-alba, a native and ornamental tree, determining the abundance and seasonal occurrence of species and the diversity of the community. Twenty three species were found belonging to 11 families, 10 of predatory habits and five phytophagous. The food habits of the eight remaining species are not known. Besides the great richness, the diversity was small, as consequence of the dominance of Lorryia formosa Cooreman, 1958 and Aculus sp., the most abundant species. These species occurred in the same microhabitat but with seasonal segregation: $L$. formosa was the most abundant in the dry season, while Aculus sp. occurred only in the rainy season. In August, when the plant lost its leaves, the following species were found in the apical branch structures: Brevipalpus sp. (aff. phoenicis), Cheletogenes sp., Euseius citrifolius Denmark \& Muma, 1970, Lorryia formosa, Pronematus sp., and diapause females of Tenuipalponychus tabebuiae Aguilar, Flechtmann \& Ochoa, 1991. In September, with leaves growing again, Aceria sp. was the only species on the plant, and its abundance decreased accentuately in October. The richness and the abundance of the mite fauna were not correlated with the temperature or the precipitation. These data suggest that the plant phenology was more important than the climate in the occurrence of species.
\end{abstract}

KEY WORDS. Acari, Bignoniaceae, biodiversity, ecology, mites, Tabebuia roseo-alba.

Muito pouco se conhece sobre os padrões de ocorrência sazonal e de diversidade de comunidades de ácaros associados a plantas, conhecimento esse, básico e imprescindível na elaboração de propostas de manejo de espécies pragas. Estudos nesse sentido foram realizados para a acarofauna associada a seringueiras cultivadas (Feres 2000, 2001, FEREs et al. 2002) e a euforbiáceas nativas herbáceas do interior desses cultivos (FERES \& NunEs 2001). Com esse objetivo e em continuidade a esses estudos, foi identificada a acarofauna associada a Tabebuia roseo-alba (Ridl.) Sand., 1954 (ipê-branco), determinando sua diversidade e a ocorrência sazonal das espécies. Essa Bignoniaceae arbórea ocorre na floresta latifoliada semidecídua dos Estados de Minas Gerais, Mato Grosso do Sul, Goiás e norte de São Paulo. Além da construção civil, é amplamente utilizada para paisagismo e no reflorestamento de terrenos secos e pedregosos, devido à sua fácil adaptação (LORENZi 2000). Nada se conhece sobre a ecologia dos ácaros associados à essa planta.

\section{MATERIAL E MÉTODOS}

A acarofauna de dois exemplares de Tabebuia roseo-alba, localizados no campus da UNESP de São José do Rio Preto $\left(20^{\circ} 47^{\prime}\right.$ $\left.03^{\prime \prime} \mathrm{S}, 49^{\circ} 21^{\prime} 36^{\prime \prime} \mathrm{W}\right)$, foi amostrada mensalmente no período de maio de 2000 a abril de 2001 . O clima da região é do tipo CwaAw de Köppen, com duas estações distintas: uma chuvosa, de outubro a março, e outra seca, de abril a setembro, que recebe apenas 15\% da precipitação total anual, que varia de $1.100 \pm 225$ a $1.250 \pm 225 \mathrm{~mm}$ (BARCHA \& ARID 1971, ARID \& BARCHA 1973).

A cada mês, foram coletadas dez folhas de cada planta, num total de 20 folhas por mês. A coleta foi realizada ao redor da copa, até a altura de $6 \mathrm{~m}$, com auxílio de podão com cabo telescópico e as folhas transportadas até o laboratório acondicionadas em sacos de polietileno. Em agosto, como as árvores encontravam-se completamente sem folhas, devido à queda natural nesse período, foi realizada a coleta de ramos para veri- 

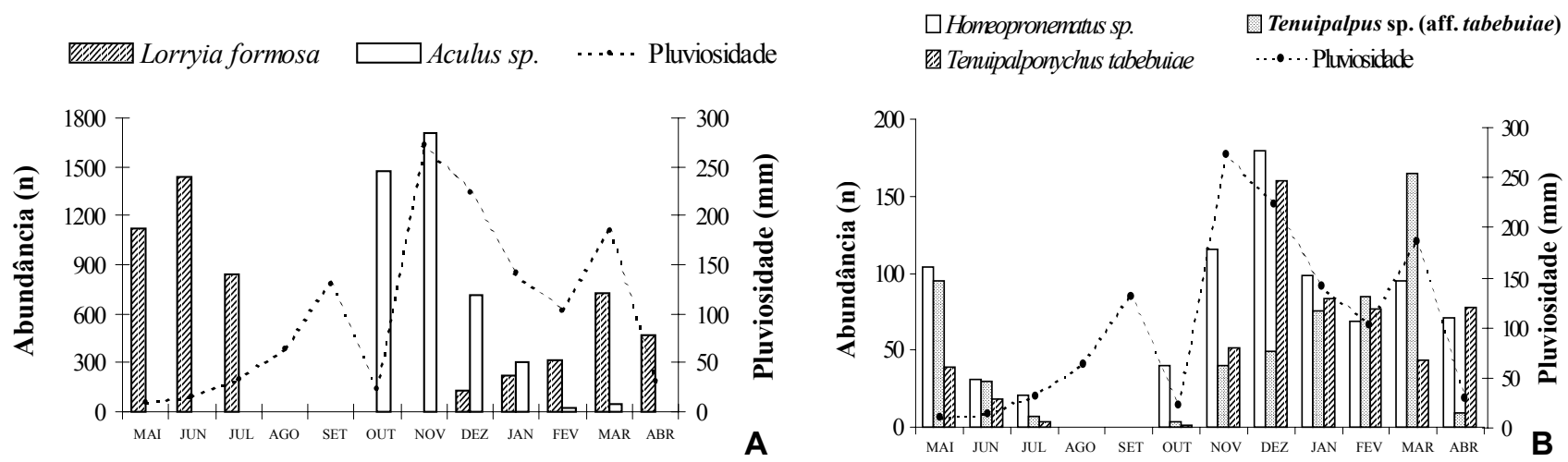

Fig. 1. Abundância mensal das cinco espécies mais abundantes em Tabebuia roseo-alba, e pluviosidade no período: (A) Lorryia formosa e Aculus sp., (B) Homeopronematus sp., Tenuipalpus sp. (aff. tabebuiae) e Tenuipalponychus tabebuiae.

ficar a ocorrência de ácaros em brácteas, gemas, flores e frutos. O material coletado foi mantido no laboratório sob refrigeração a cerca de $10^{\circ} \mathrm{C}$ por, no máximo, uma semana. Este procedimento não só proporciona melhor conservação do material, como também diminui o metabolismo dos ácaros coletados, reduzindo seu deslocamento.

A triagem foi feita sob microscópio estereoscópico, sendo coletados todos os ácaros encontrados em um dos folíolos de cada folha. Os ácaros foram montados em lâminas de microscopia com meio de Hoyer (FlechtMAnN 1975), e a identificação e contagem dos espécimes foi feita sob microscópio óptico com contraste de fase.

A diversidade e a uniformidade da acarofauna de Tabebuia roseo-alba foram calculadas pelos índices de Shannon-Wiener $\left(\mathrm{H}^{\prime}\right)$ e de Pielou (e), respectivamente (Odum 1988). A diversidade teórica máxima (H' max.) foi determinada segundo KreBs (1999). Para cada espécie foram determinadas, além da abundância, a constância e a ocorrência sazonal. A constância (c) foi calculada segundo DAJOz (1973), sendo as espécies classificadas como constantes (c $>50 \%)$, acessórias $(25<\mathrm{c}<50 \%)$ e acidentais (c $<25 \%)$.

A influência dos parâmetros climáticos sobre as espécies mais abundantes, foi verificada pela aplicação do índice de correlação linear de Pearson (ZAR 1999). O mesmo índice foi aplicado para verificar a influência do clima sobre a riqueza e abundância total de ácaros da comunidade. Os dados de temperatura e pluviosidade foram obtidos junto à Coordenadoria de Assistência Técnica Integral (CATI), de São José do Rio Preto, São Paulo; o nível de significância adotado foi de $5 \%(\mathrm{p}<0,05)$.

O grau de similaridade entre as espécies, com relação a abundância e ocorrência sazonal, foi determinado pelo índice de similaridade de Morisita-Horn (KREBs 1999), com posterior análise de agrupamento pelo método de média não ponderada (UPGMA). Foram consideradas agrupadas as espécies com similaridade igual ou superior a $83 \%$.

\section{RESULTADOS}

Foram coletados 11.884 ácaros pertencentes a 23 espécies de 21 gêneros e 11 famílias. Das 15 espécies com hábito alimen- tar conhecido, 10 são predadoras ou preponderantemente predadoras e cinco são fitófagas (Tab. I).

As espécies mais abundantes foram Lorryia formosa Cooreman, 1958 e Aculus sp., fitófagos que representaram 80,2\% da abundância total. Ambas as espécies ocuparam o mesmo microhábitat, na página inferior das folhas, preponderantemente na junção da nervura central com as secundárias. No entanto, diferiram quanto à ocorrência sazonal: a primeira ocorreu ao longo do ano, com maior abundância na estação seca, enquanto Aculus sp. ocorreu apenas na estação chuvosa (Fig. 1A). Alguns indivíduos de ambas as espécies foram encontrados na página superior ou no pecíolo. Esse fato ocorreu tanto nos meses de maior abundância populacional de cada espécie (Aculus sp.: outubro e novembro, L. formosa: maio, junho e julho), como nos meses em que uma delas foi muito mais abundante que a outra (Aculus sp.: outubro e novembro, Lorryia formosa: março) (Tab. I).

Homeopronematus sp., Tenuipalpus sp. (aff. tabebuiae) e Tenuipalponychus tabebuiae Aguilar, Flechtmann \& Ochoa 1991, ocorreram preponderantemente na estação chuvosa e, juntas, representaram 16,3\% da abundância total (Tab. I, Fig. 1B). As 18 espécies restantes corresponderam, juntas, a apenas 3,5\% da abundância total.

Em agosto, quando a planta perdeu suas folhas devido à queda sazonal, exemplares de Lorryia formosa, Euseius citrifolius, Pronematus sp., Cheletogenes sp., Brevipalpus sp. (aff. phoenicis) e fêmeas de Tenuipalponychus tabebuiae foram encontrados nas estruturas dos ápices dos ramos (Tab. I). As fêmeas de Tenuipalponychus tabebuiae encontravam-se em diapausa, abrigadas na face interna da base das brácteas. Aceria sp. foi a única espécie que apresentou pico populacional em setembro (Tab. I), mês de reenfolhamento da planta, quando $90,3 \%$ do total de espécimens foram coletados.

A maioria das espécies (39\%) foi acidental e apenas sete espécies (30,5\%) ocorreram por mais de sete meses, sendo consideradas constantes. A abundância e a riqueza da acarofauna apresentaram dois picos: um coincidente, no início da estação chuvosa e outro defasado, com a maior riqueza no início da estação seca (março/abril) e a maior abundância em plena estação seca (maio/junho), como conseqüência da explosão populacional de Lorryia formosa (Tab. I, Fig. 2). A abundância e

Revista Brasileira de Zoologia 20 (3): 373-378, setembro 2003 
Tabela I. Abundância mensal e total, hábito alimentar e constância de ocorrência das 23 espécies registradas em Tabebuia roseo-alba.

\begin{tabular}{|c|c|c|c|c|c|c|c|c|c|c|c|c|c|c|c|}
\hline Espécies & Hábito & Mai & Jun & Jul & Ago* & Set & Out & Nov & Dez & Jan & $\mathrm{Fev}$ & Mar & Abr & Total & Constância \\
\hline \multicolumn{16}{|l|}{ Bdellidae } \\
\hline Spinibdella sp. & $P$ & - & - & - & - & - & - & 1 & - & - & - & 1 & - & 2 & Acidental \\
\hline \multicolumn{16}{|l|}{ Cheyletidae } \\
\hline Cheletogenes sp. & $P$ & 1 & - & - & * & - & 1 & - & - & - & - & - & - & 2 & Acidental \\
\hline Chiapacheylus sp. & $P$ & - & - & - & - & - & - & - & - & - & - & - & 3 & 3 & Acidental \\
\hline \multicolumn{16}{|l|}{ Eriophyidae } \\
\hline Aceria sp. & $\mathrm{F}$ & - & - & - & - & 130 & 13 & 1 & - & - & - & - & - & 144 & Acessória \\
\hline Aculus sp. & $\mathrm{F}$ & - & - & - & - & - & 1475 & 1707 & 708 & 305 & 21 & 50 & - & 4266 & Acessória \\
\hline \multicolumn{16}{|l|}{ Malaconothridae } \\
\hline Trimalaconothrus sp. & $?$ & - & - & - & - & - & - & - & - & - & - & 1 & - & 1 & Acidental \\
\hline \multicolumn{16}{|l|}{ Phytoseiidae } \\
\hline Euseius citrifolius & PP & 4 & - & 4 & * & 1 & 28 & 12 & 9 & 4 & - & 2 & - & 64 & Constante \\
\hline Euseius sibelius & PP & - & - & 1 & - & - & - & - & 1 & 1 & 1 & 1 & 5 & 10 & Acessória \\
\hline Galendromus. annectens & PP & - & - & - & - & - & - & - & - & - & 1 & 1 & 1 & 3 & Acessória \\
\hline Metaseiulus sp. (cf. cornus) & PP & 2 & 2 & 1 & - & - & 1 & 7 & 3 & - & - & - & 8 & 24 & Constante \\
\hline Typhlodromus sp. & PP & - & - & - & - & - & - & 1 & - & 1 & - & 9 & - & 11 & Acessória \\
\hline \multicolumn{16}{|l|}{ Stigmaeidae } \\
\hline Agistemus sp. & $P$ & 1 & 10 & 3 & - & - & 10 & 6 & 1 & 7 & 7 & 16 & 3 & 64 & Constante \\
\hline Zetzellia sp. & $P$ & - & - & - & - & - & 1 & - & - & - & 1 & - & - & 2 & Acidental \\
\hline \multicolumn{16}{|l|}{ Tarsonemidae } \\
\hline Fungitarsonemus sp. & $\mathrm{M} ?^{1}$ & - & - & - & - & - & - & - & - & - & - & - & 1 & 1 & Acidental \\
\hline \multicolumn{16}{|l|}{ Tenuipalpidae } \\
\hline Brevipalpus sp. (aff. phoenicis) & $\mathrm{F}$ & - & 1 & - & * & - & - & 1 & 1 & 5 & - & 5 & 5 & 18 & Acessória \\
\hline Tenuipalpus sp. (aff. tabebuiae) & $\mathrm{F}$ & 95 & 30 & 7 & - & - & 4 & 40 & 49 & 76 & 85 & 165 & 9 & 560 & Constante \\
\hline \multicolumn{16}{|l|}{ Tetranychidae } \\
\hline Tenuipalponychus tabebuiae & $\mathrm{F}$ & 39 & 18 & 4 & * & 1 & 1 & 52 & 160 & 84 & 77 & 44 & 78 & 558 & Constante \\
\hline \multicolumn{16}{|l|}{ Tydeidae } \\
\hline Homeopronematus anconai & $P ?^{2}$ & - & - & - & - & - & 23 & - & - & - & - & - & - & 23 & Acidental \\
\hline Homeopronematus sp. & $\mathrm{P} ?^{2}$ & 104 & 31 & 21 & - & - & 40 & 115 & 180 & 98 & 69 & 95 & 71 & 824 & Constante \\
\hline Lorryia formosa & $F ?^{3}$ & 1121 & 1442 & 839 & * & - & 3 & 4 & 126 & 226 & 316 & 722 & 466 & 5265 & Constante \\
\hline Pausia sp. & $P ?^{2}$ & - & - & - & - & - & 2 & 9 & - & - & - & - & - & 11 & Acidental \\
\hline Pronematus sp. & $\mathrm{P} ?^{2}$ & 1 & 1 & 1 & * & - & 7 & - & - & - & - & - & - & 10 & Acessória \\
\hline \multicolumn{16}{|l|}{ Winterschmidtiidae } \\
\hline Czenspinskia sp. & $M ?^{4}$ & - & - & - & - & - & - & - & - & - & - & 17 & 1 & 18 & Acidental \\
\hline Abundância & & 1368 & 1535 & 881 & - & 132 & 1609 & 1956 & 1238 & 807 & 578 & 1129 & 651 & 11884 & \\
\hline Riqueza & & 9 & 8 & 9 & - & 3 & 14 & 13 & 10 & 10 & 9 & 14 & 12 & & \\
\hline
\end{tabular}


A

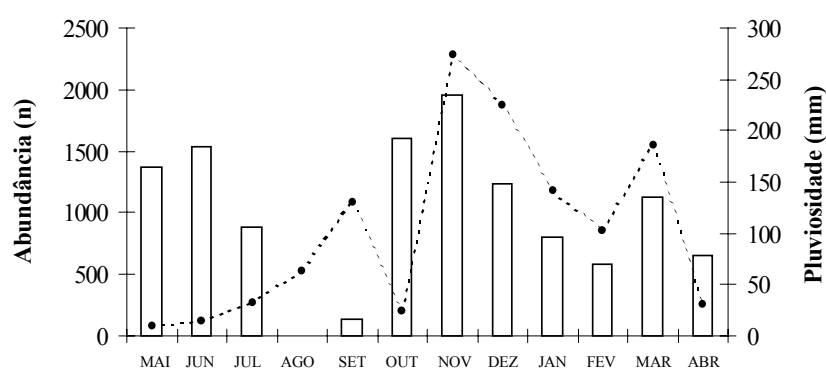

B

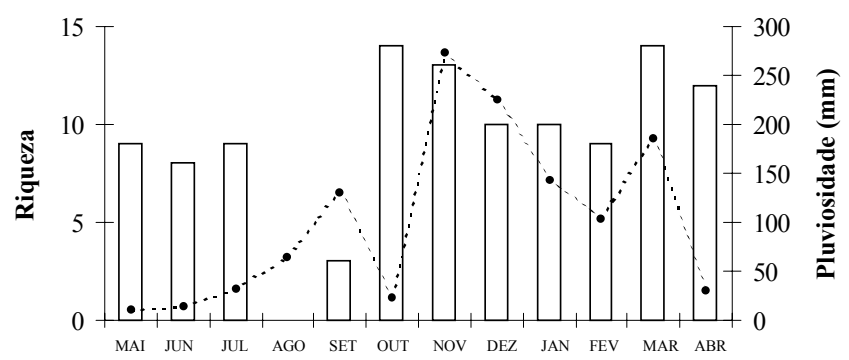

Fig. 2. Abundância (A) e riqueza (B) mensais da acarofauna de Tabebuia roseo-alba, e pluviosidade no período.

a riqueza da acarofauna não foram correlacionadas com os parâmetros climáticos, mas a ocorrência de Homeopronematus sp. foi positivamente correlacionada com a precipitação pluviométrica, enquanto a de $L$. formosa foi negativamente correlacionada com a temperatura (Tab. II).

Tabela II. Correlação entre a abundância mensal das cinco espécies de ácaros mais abundantes, a abundância total e riqueza da acarofauna com a temperatura e a pluviosidade mensal.

\begin{tabular}{lcc}
\multicolumn{1}{c}{ Espécies } & Temperatura & Pluviosidade \\
\hline Lorryia formosa & $-0,59^{*}$ & $-0,50$ \\
Aculus sp. & 0,46 & 0,46 \\
Homeopronematus sp. & 0,44 & $0,59^{*}$ \\
Tenuipalpus sp. (aff. tabebuiae) & 0,29 & 0,33 \\
Tenuipalponychus tabebuiae & 0,49 & 0,51 \\
\hline \multicolumn{1}{c}{ Comunidade } & Temperatura & Pluviosidade \\
\hline Abundância total & 0,12 & 0,19 \\
Riqueza de espécies & 0,48 & 0,22 \\
\hline
\end{tabular}

* Valores significativos para $\mathrm{P}<0,05$

Apesar da grande riqueza (23 espécies), a diversidade foi baixa $\left(\mathrm{H}^{\prime}=0,6\right)$, alcançando cerca de metade da diversidade máxima teórica $\left(\mathrm{H}^{\prime}=1,32\right)$, como conseqüência da baixa uniformidade $(e=0,45)$.

A análise de similaridade evidenciou seis agrupamentos de espécies, de acordo com os períodos de maior abundância populacional (Fig. 3): I) Tenuipalpus sp. (aff. tabebuiae), Agistemus sp., Brevipalpus sp. (aff. phoenicis), que foram mais abundantes em março; II) Tenuipalponychus tabebuiae e Homeopronematus sp., espécies constantes e mais abundantes em dezembro; III) Typhlodromus sp. e Czenspinskia sp., espécies pouco abundantes, com pico populacional em março; IV) Chiapacheylus sp., Trimalaconothrus sp. e Fungitarsonemus sp. (DeLeon, 1962), espécies raras, que ocorreram apenas em março ou abril; V) Homeopronematus anconai Baker (1943) e Pronematus sp., que foram mais abundantes em outubro; VI) Aculus sp. e Euseius citrifolius, que foram mais abundantes em outubro e novembro.

\section{DISCUSSÃO}

Apesar da grande riqueza de espécies de ácaros associados a Tabebuia roseo-alba, a diversidade foi baixa, com dominância de duas espécies. Esses resultados estão de acordo com a proposição de ODum (1988) que, em comunidades de regiões tropicais sazonais poucas espécies são muito abundantes e, portanto, dominantes na comunidade, gerando baixa uniformidade. As cinco espécies mais abundantes ocorreram preponderantemente na página inferior dos folíolos, onde ficaram abrigadas do efeito mecânico das chuvas, que pode remover parte dos indivíduos das espécies que ocorrem na página superior. Duas dessas espécies, Aculus sp. e Lorryia formosa, alcançaram densidades muito altas o que, provavelmente, provocou o deslocamento de exemplares, da mesma ou da espécie menos abundante, para a página superior ou pecíolo das folhas.

Das espécies encontradas nas estruturas dos ápices dos ramos (brácteas, gemas etc.), durante o período de desfolha natural, Tenuipalponychus tabebuiae e Aceria sp., apresentaram ciclo de vida associado à fenologia da planta. A primeira entrou em diapausa na época de ausência de folhas (Feres \& Bellini 2002), voltando à atividade logo após o reenfolhamento. Já a ocorrência de Aceria sp. foi associada ao período de reenfolhamento da planta. Durante o envelhecimento das folhas ocorre translocação de nutrientes para o desenvolvimento de ramos e folhas novas, aumentando sua concentração nessas estruturas (BEEvers 1976 apud White 1984, Hill 1980). Além disso, folhas jovens possuem diferenças na concentração de componentes químicos quando comparadas com folhas mais velhas (WendLer et al. 1995, VALJAKKa et al. 1999), o que pode ter limitado a ocorrência sazonal de Aceria sp. ao período em que a planta apresentava folhas jovens.

O grande número de agrupamentos, evidenciados na análise da ocorrência sazonal das espécies acarinas associadas a T. roseo-alba, indica que não houve um padrão quanto ao período de ocorrência. Reforça essa sugestão, o fato que apenas duas das cinco espécies mais abundantes tiveram sua ocorrência correlacionada com um dos parâmetros climáticos. Além disso, nem a riqueza nem a abundância total foram correlacionadas com o clima. Essa ausência de padrão, provavelmente, é reflexo da grande variedade de hábitos alimentares e de microhabitats ocupados. Assim, a ocorrência de ácaros em T. roseo-alba foi mais influenciada pela fenologia da planta, que limitou a ocorrência das espécies em agosto e setembro, época de ausência de folhas e reenfolhamento, respectivamente, que pelo clima. 


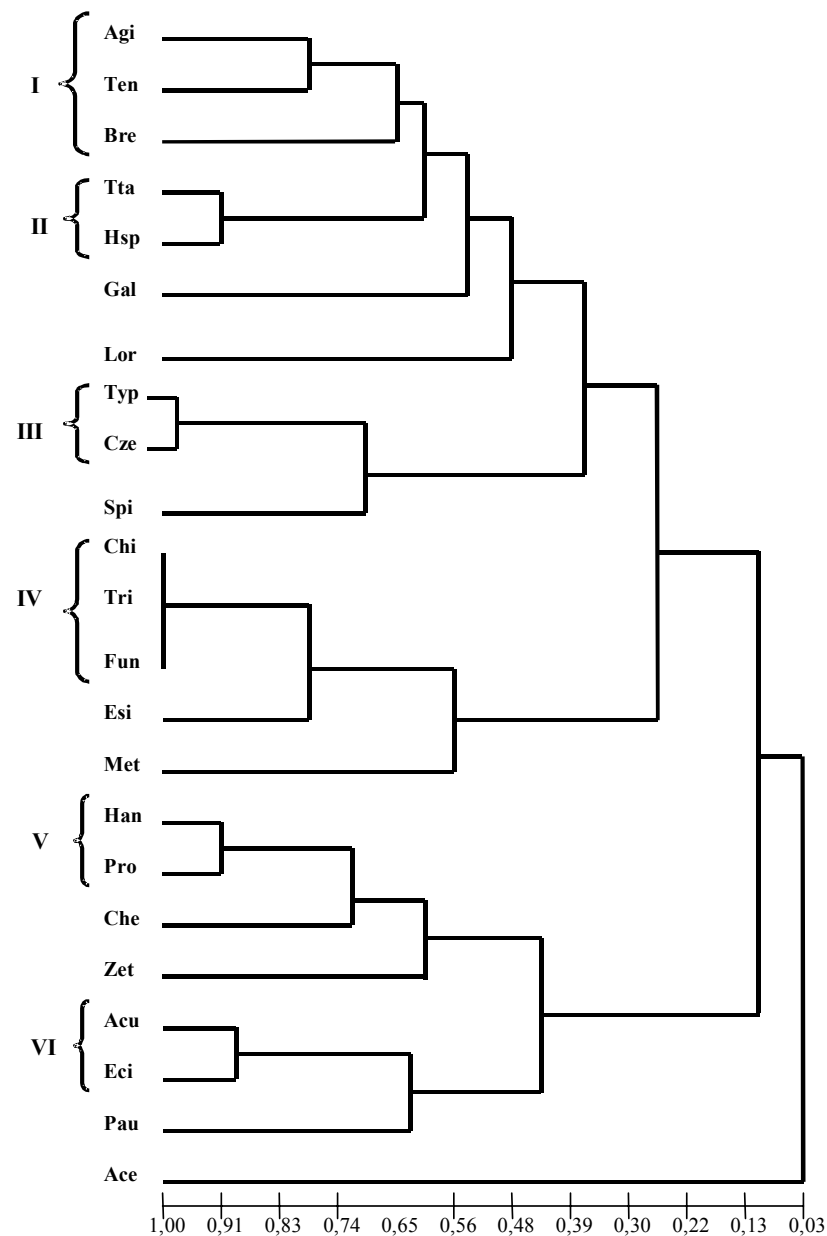

Fig. 3. Dendrograma resultante de análise de agrupamento, na matriz do índice de similaridade de Morisita-Horn, evidenciando os seis agrupamentos de espécies com similaridade maior que $83 \%$ na abundância mensal. (Ace) Aceria sp., (Acu) Aculus sp., (Agi) Agistemus sp., (Bre) Brevipalpus sp. (aff. phoenicis), (Che) Cheletogenes sp., (Chi) Chiapacheylus sp., (Cze) Czenspinskia sp., (Eci) Euseius citrifolius, (Esi) Euseius sibelius, (Fun) Fungitarsonemus sp., (Gal) Galendromus annectens, (Han) Homeopronematus anconai, (Hsp) Homeopronematus sp., (Lor) Lorryia formosa, (Met) Metaseiulus sp. (cf. cornus), (Pau) Pausia sp., (Pro) Pronematus sp., (Spi) Spinibdella sp., (Ten) Tenuipalpus sp. (aff. tabebuiae) (Tta) Tenuipalponychus tabebuiae, (Tri) Trimalaconothrus sp., (Typ) Typhlodromus sp., (Zet) Zetzellia sp.

\section{AGRADECIMENTOS}

Ao Dr. Mauricio Sergio Zacarias, Pesquisador-CNPq, Epamig-CTSM/EcoCentro, Lavras e ao MSc. Anibal Ramadan Oliveira, Programa de Pós-graduação em Ciências- Zoologia, Departamento de Zoologia, Instituto de Biociências, USP, pela identificação dos Tydeidae e Malaconothridae, respectivamente. Ao Núcleo de Produção de Sementes de São José do Rio Preto, Coordenadoria de Assistência Técnica Integral (CATI), da
Secretaria de Agricultura e Abastecimento, São Paulo, pelo fornecimento dos dados climáticos.

\section{REFERÊNCIAS BIBLIOGRÁFICAS}

ARID, F.M. \& S.F. BARCHA. 1973. Água Subterrânea na formação Bauru- Região Norte Ocidental do Estado de São Paulo. Boletim de Ciências da Faculdade de Filosofia, Ciências e Letras de São José do Rio Preto. 1: 67-101.

BAKER, E.W. 1965. A review of the genera of the Family Tydeidae (acarina). Advances in Acarology, New York, 2: 95-133.

BaKer, E.W. \& A. E. Wharton. 1952. An introduction to Acarology. New York, MacMillam Company, 465p.

BARCHA, S.F. \& F.M. ARID. 1971. Estudo da evapotranspiração na região Norte Ocidental do Estado de São Paulo. Revista de Ciências da Faculdade de Ciências e Letras de Votuporanga 1: 99-122.

BAYAN, A. 1986. Tydeid mites associated with aplles in Lebanon (Acari:Actinedida: Tydeidae). Acarologia, Paris, 27 (4): 311316.

Calvert, D. J. \& C. B. Huffaker. 1974. Predator (Metaseiulus occidentalis) - prey (Pronematus spp.) Interactions under sulfur and cattail pollen applications in a noncommercial vineyard. Entomophaga, Paris, 19 (3): 361-369.

DAJOz, R. 1973. Ecologia Geral. Rio de Janeiro, Vozes-Edusp, $472 \mathrm{p}$.

FERES, R.J.F. 2000. Levantamento e observações naturalísticas da acarofauna (Acari: Arachnida) de seringueiras cultivadas (Hevea spp., Euphorbiaceae) no Brasil. Revista Brasileira de Zoologia, Curitiba, 17 (1): 157-173.

. 2001 [2002]. Ácaros eriofiídeos (Acari: Eriophyidae) em Seringueira (Hevea brasiliensis Muell. Arg.: Euphorbiaceae) no Brasil, p. 31-36. In: L.A.N. DE SÁ, \& G.J. DE MoraES (Orgs). Ácaros de Importância Quarentenária. Jaguariúna, Embrapa Meio Ambiente, 40p.

Feres, R.J.F. \& M.A. NunEs. 2001. Ácaros (Acari, Arachnida) associados a euforbiáceas nativas em áreas de cultivo de seringueiras (Hevea brasiliensis Muell. Arg., Euphorbiaceae) na região noroeste do Estado de São Paulo, Brasil. Revista Brasileira de Zoologia, Curitiba, 18 (4): 1253-1264.

Feres, R.J.F. \& M.R. BelLini. 2002. Ocorrência de diapausa em Tenuipalponychus tabebuiae Aguilar, Flechtmann \& Ochoa 1991 (Acari, Tetranychidae) no Estado de São Paulo, Brasil. Revista Brasileira de Zoologia, Curitiba, 19 (Supl. 2): 219224.

Feres, R.J.F., D. De C. Rossa-Feres, R.D. Daud \& R.S. Santos. 2002. Diversidade de ácaros (Acari, Arachnida) em seringueiras (Hevea brasiliensis Muell. Arg., Euphorbiaceae) na região noroeste do Estado de São Paulo, Brasil. Revista Brasileira de Zoologia, Curitiba, 19 (1): 137-144.

Flechtmann, C.H.W. 1973. Lorryia formosa Cooreman, 1958 Um ácaro dos citros pouco conhecido no Brasil. Ciência e Cultura, São Paulo, 25 (12): 1179-1181. $344 \mathrm{p}$

. 1986. Ácaros em produtos armazenados e na poeira domiciliar. Piracicaba, Fundação de Estudos Agrários "Luiz de Queiroz"- FEALQ, 67p.

Flechtmann, C.H.W. \& R.J. Arleu. 1984. Oligonychus coffeae (Nietner, 1861), um ácaro tetraniquídeo da seringueira 
(Hevea brasiliensis) novo para o Brasil e observações sobre outros ácaros desta planta. Ecossistema, Espírito Santo do Pinhal, 9: 123-125.

Gerson, U. 1968. Five tydeid mites from Israel (Acarina: Prostigmata). Israel Journal of Zoology, Jerusalem, 17: 191-198.

HiLL, J. 1980. The remobilization of nutrients from leaves. Journal of Plant Nutrition, New York, 2: 407-444.

Jeppson, L.R., H.H. KeIfer, \& E.W. BAKer. 1975 - Mites injurious to economic plants. Berkeley, University of California Press, $614 \mathrm{p}$.

KrantZ, G.W.1978. A manual of Acarology. Corvallis, Oregon State University Book Stores, 509p.

Krebs, C.J. 1999. Ecological Methodology. Menlo Park, Ed. Addison Wesley Longman Inc., 620p.

LINDQUIST, E.E. 1986. The world genera of Tarsonemidae (Acari: Heterostigmata): a morphological, phylogenetic and systematic revision, with a reclassification of family-group taxa in the Heterostigmata. Memoirs of Entomological Society of Canada, Ottawa, (136): 1-517.

LORENZI, H. 2000. Árvores brasileiras. Manual de Identificação e cultivo de plantas arbóreas nativas do Brasil. Nova Odessa, Instituto Plantarum, vol. 1, 367p.

McCoy, C. W.; A. G. Selhime \& R. F. Kanavel. 1967. The feeding behavior and biology of Parapronematus acaciae (Acarina: Tydeidae). Florida Entomologist, Winter Haven, 52 (1): 1319.

Recebido em 09.I.2003; aceito em 15.V.2003.
McMurtry, J.A. \& B.A. Croft. 1997. Life-styles of phytoseiid mites and their roles in biological control. Annual Review of Entomology, Palo Alto, 42: 291-321.

Odum, E.P. 1988. Ecologia. Rio de Janeiro, Editora Guanabara, 432p.

SMIRNOFF, W.A. 1957. An undescribed species of Lorryia sp. (Acarina: Tydeidae) causing injury to citrus trees in Marrocco. Journal of Economic Entomology, Lanham, 50 (3): 361362.

ValjakKa, M.; E-M. Luomala; J. Kangasjärvi \& E. VapaAvuori. 1999. Expression of photosynthesis- and senescence- related genes during leaf development and senescence in silver birch (Betula pendula) seedlings. Physiologia Plantarum, Lund, 106: 302-310.

Wendler, R.; P.O. Carvalho; J.S. Pereira \& P. Millard. 1995. Role of nitrogen remobilization from old leaves for new leaf growth of Eucalyptus globulus seedlings. Tree Physiology, Victoria, 15: 679-783.

WHITE, T.C.R. 1984. The abundance of invertebrate herbivores in relation to the availability of nitrogen in stressed food plants. Oecologia, Berlin, 63: 90-105.

Woolley, T.A. 1988. Acarology: mites and human welfare. Fort Collins, Colorado, Library of Congress cataloging in Publication, 484p.

ZAR, J.H. 1999. Biostatistical analysis. New Jersey, Prentice Hall, $663 p$.

Revista Brasileira de Zoologia 20 (3): 373-378, setembro 2003 